\title{
X-ray polarization fluctuations induced by cloud eclipses in active galactic nuclei
}

\author{
F. Marin and M. Dovčiak
}

\author{
Astronomical Institute of the Academy of Sciences, Bočni II 1401, 14131 Prague, Czech Republic \\ e-mail: frederic.marin@asu.cas.cz
}

Received 26 August 2014 / Accepted 31 October 2014

\begin{abstract}
Context. A fraction of active galactic nuclei (AGN) show dramatic X-ray spectral changes on the day-to-week time scales associated with variation in the line of sight of the cold absorber.

Aims. We intend to model the polarization fluctuations arising from an obscuration event, thereby offering a method of determining whether flux variations are due to occultation or extreme intrinsic emission variability.

Methods. Undertaking 1-100 keV polarimetric simulations with the Monte Carlo code Stokes, we simulated the journey of a variety of cold gas clouds in front of an extended primary source. We varied the hydrogen column density $n_{\mathrm{H}}$ and size of the absorber, as well as the initial polarization state of the emitting source, to cover a wide range of scenarios.

Results. Simulations indicate that different results are expected according to the initial polarization of the extended continuum source. For unpolarized primary fluxes, large $\left(\sim 50^{\circ}\right)$ variations of the polarization position angle $\psi$ are expected before and after an occultation event, which is associated with very low residual polarization degrees $(P \ll 1 \%)$. In the case of an emitting disk with intrinsic, position-independent polarization, and for a given range of parameters, X-ray eclipses significantly alter the observed polarization spectra, with most of the variations seen in $\psi$. Finally, non-uniformly polarized emitting regions produce very distinctive polarization variations due to the successive covering and uncovering of different portions of the disk. Plotted against time, variations in $P$ and $\psi$ form detectable P Cygni type profiles that are distinctive signatures of non-axisymmetric emission.

Conclusions. We find that X-ray polarimetry is particularly adapted to probing X-ray eclipses due to Compton-thin and Comptonthick gas clouds. Polarization measurements would distinguish between intrinsic intensity fluctuations and external eclipsing events, constrain the geometry of the covering medium, and test the hypothesis of non-uniformly emitting disks predicted by general relativity.
\end{abstract}

Key words. galaxies: Seyfert - polarization - radiative transfer - relativistic processes - scattering - X-rays: general

\section{Introduction}

Since the beginning of the millennium, the number count of active galactic nuclei (AGN) known as "changing look" Seyfert galaxies is increasing. More than a dozen examples present $\mathrm{X}$-ray fluxes and hardness ratio light curves exhibiting large variabilities, up to a factor 3 , on year, week, or even day time scales (Risaliti et al. 2002). Such a variability pattern is attributed either to intrinsic vacillation of the nucleus or to transition from reflection-dominated (Compton-thick, $n_{\mathrm{H}} \geq 10^{24} \mathrm{~cm}^{-2}$ ) to transmission-dominated (Compton-thin, $n_{\mathrm{H}} \leq 10^{23} \mathrm{~cm}^{-2}$ ) AGN states because of an occultation event along the observer's line of sight (Risaliti 2010). In this picture, discrete absorbing clouds orbit the central supermassive black hole and its surrounding emitting accretion disk, and partially or fully cover the central region.

From photo-ionization, $n_{\mathrm{H}}$ and timing arguments, Risaliti et al. (2002) estimate that the absorbing material has to be clumpy and closer to the central engine than the so-called obscuring torus situated along the AGN equatorial plane and responsible for the observational dichotomy between type-1 (poleon) and type-2 (edge-on) quasars. According to the authors, the reservoir of obscuring clouds could be associated with the low-ionization line broad line region (LIL BLR), where neutral or mildly ionized gas with $n_{\mathrm{H}}$ on the order of $10^{22-25} \mathrm{~cm}^{-2}$ are in Keplerian motion (Markowitz et al. 2014). Such a picture is consistent with the suggestion of Netzer \& Laor (1993) and supported by the reverberation mapping observations of Suganuma et al. (2006), implying that the LIL BLR coincides with the dust sublimation radius of the circumnuclear torus. Observations so far point toward a distance of the eclipsing gas cloud on the order of $10^{13-16} \mathrm{~cm}$ with number densities $10^{8-11} \mathrm{~cm}^{-3}$ (Risaliti et al. 2009; Markowitz et al. 2014), velocities of a few thousand kilometers per second (Risaliti et al. 2009) and a size of the emitting X-ray source lower than $10^{13} \mathrm{~cm}$ (Risaliti et al. 2009).

Occultation from a spherical, homogeneous cloud not only alters the X-ray light curve of obscured objects, but is also expected to affect their observed spectroscopic and polarimetric properties. Risaliti et al. (2011a) explored the successive shading of the receding and approaching parts of an AGN accretion disk, and show that an X-ray eclipse would affect the shape of the line profile and change the fluxes between the two halves of the disk owing to Doppler boosting. They also estimated that a $50 \mathrm{ks} X M M-N e w t o n$ or Suzaku observation could detect the asymmetries in the X-ray spectra at different obscuring phases of the AGN disk. When scaling down in mass, past studies of dipping ${ }^{1}$ low-mass X-ray binaries (LMXRB) and magnetic cataclysmic variables $(\mathrm{mCV})$ have explored how occultation and

\footnotetext{
1 Dipping LMXRB are a subclass of X-ray binaries (XRB) known to show periodic dips in their X-ray light curves. Those dips are associated with obscuration by a thick gas stream located at the outer edge of the accretion disk, flowing from the companion star to the compact object.
} 

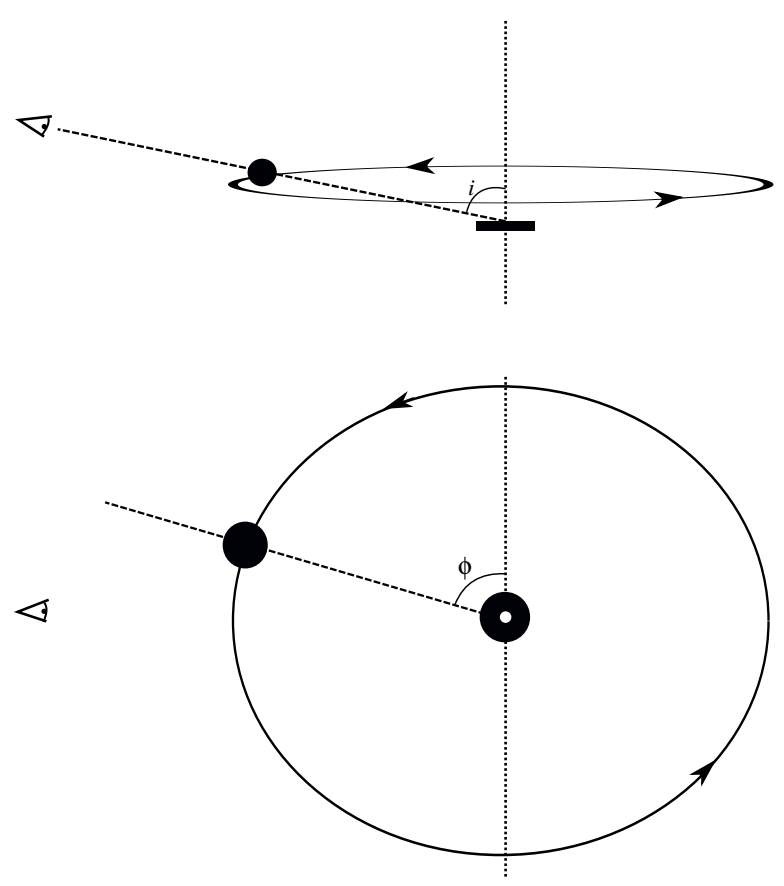

Fig. 1. Representative sketch of a Keplerian cloud rotating around a central, extended source. The obscuring region moves from $\phi=0^{\circ}$ to $180^{\circ}$, with a maximum obscuration of the source at $\phi=90^{\circ}$. The observer's viewing angle $i$ is set to $50^{\circ}$. The model is seen from edge-on (top figure) and pole-on (bottom) inclinations.

absorption effects from the accretion stream structure affect the observed spectroscopic properties (White \& Holt 1982). James et al. (2002) derived the inclination and white dwarf mass of the $\mathrm{mCV}$ PQ Gem coherently with optical polarization data. However, a polarimetric observation in only one and different (the optical) band is not enough for a complete interpretation, so X-ray polarimetry is needed to confirm those results. Polarization is expected to rise since the central system, either obscured by a passing cloud at an intermediate AGN inclination or by the accretion stream in XRB, becomes less symmetric and offers new scattering targets.

Therefore, our aim is to present the first radiative transfer study of X-ray occultation events through the prism of X-ray polarimetry. By doing so, we want to stress the necessity for a new X-ray polarimetric mission in order to assess some relevant questions in the field of AGN variability, (Are flux variations due to occultation or extreme intrinsic emission variability? Is the accretion disk emitting uniformly or, according to general relativity, is the emission spatially dependent?). We therefore explore the impact of a single, spherical cloud of neutral gas eclipsing an extended source that is representative of the irradiating accretion disk. We present in Sect. 2 a variety of investigations, going through a large parameter space (energy band, hydrogen column density, size of the occulting region, intrinsic polarization level, and spatial location). We discuss our modeling results in Sect. 3 and present our conclusions in Sect. 4.

\section{Signatures of isolated X-ray occultation}

The physical origin of AGN X-ray light curve variations is uncertain. Several mechanisms with different physical time scales seem to superimpose: accretion-disk instabilities, variable accretion rates, variable obscuration of the nuclear source and/or microlensing due to stars in the host galaxy (see the reviews by
Ulrich et al. 1997; Peterson 2001). Since starlight is expected to show no polarization in the X-ray range (Dolan 1967, see Sect. 3), most of the potential polarization comes from the disk and cloud obscuration. In this paper, we thus vary the polarization of the disk and explore the polarization variations due to cloud obscuration to investigate if and when the polarization signal is dominated by intrinsic emission rather than the intervening clouds.

\subsection{X-ray polarization modeling with Stokes}

Radiative transfer calculations are computed using the Monte Carlo code Stokes (Goosmann \& Gaskell 2007; Marin et al. 2012), which has already been used to compute the polarization emerging from a clumpy distribution of gas around the central engine of NGC 1365 (Marin et al. 2013). The code simulates the radiative transfer between user-defined emitting and reprocessing media, computes polarization induced by multiple reprocessing events, and stores the final polarization properties as a function of the observer's inclination and azimuthal angle. Stokes gets its name from the Stokes formalism used to quantify the polarization state of each photon $\left(S=(I, Q, U, V)^{\mathrm{T}}\right)$. In this formalism, the polarization degree $P$, ranging from $0 \%$ (depolarized) to $100 \%$ (fully polarized), is calculated with

$P=\frac{\sqrt{Q^{2}+U^{2}+V^{2}}}{I}$,

and the linear polarization position angle $\psi$, relative to the two chosen orthogonal bases of the polarization ellipse, is

$\psi=\frac{1}{2} \tan ^{-1}\left(\frac{U}{Q}\right)$

Radiation works its way through the model region and may undergo scattering, absorption, or re-emission events. The algorithms for inelastic Compton scattering onto bound and free electrons, photo-ionization, and recombination effects are implemented in the X-ray regime. The emission directions, mean free paths between scattering events, and the reprocessing angles are computed by Monte Carlo routines based on classical intensity distributions. Mueller matrices are used to evaluate the change in polarization after each scattering event. Photo-absorption above the iron $\mathrm{K}$-shell and the subsequent emission of iron $\mathrm{K} \alpha$ or $\mathrm{K} \beta$ line photons is included and weighted against the probability of Auger effects. The scattering phase function, the polarization state, and the emission probability of the re-emitted photon are computed from quantum mechanics (Lee et al. 1994; Lee 1994). Stokes was tested against the spectropolarimetric modeling of AGN tori and found to be consistent (see discussion in Goosmann \& Matt 2011). For further details about the code, please refer to the complete description of the polarization properties and transformation of radiation during scattering events described in Goosmann \& Gaskell (2007) and references therein.

For the remainder of this paper, our fixed baseline model consists of a purely emitting, extended accretion disk with a radius $R$ of $10^{13} \mathrm{~cm}$. The disk has a slab geometry with height $h \ll R$ and radiates $>10^{9}$ input photons according to a powerlaw shape $F_{*} \propto v^{-\alpha}$ with a typical AGN spectral index $\alpha=1$. At a distance of $10^{15} \mathrm{~cm}$, an atomic molecular cloud filled with neutral matter rotates in Keplerian motion around the source with an orbital velocity of $4000 \mathrm{~km} \mathrm{~s}^{-1}$. The matter of the obscuring cloud, which is assumed to be cold (i.e. $T<10^{6} \mathrm{~K}$ ), consists of neutral $\mathrm{H}, \mathrm{He}, \mathrm{C}, \mathrm{N}, \mathrm{O}, \mathrm{Ne}, \mathrm{Na}, \mathrm{Mg}, \mathrm{Al}, \mathrm{Si}, \mathrm{S}, \mathrm{Ar}, \mathrm{Ca}, \mathrm{Fe}$, and 
Ni elements with cosmic abundances. The electron optical depth of the cloud is fixed to unity (as may be assumed for LIL BLR clouds, Wills et al. 1999). The inclination of the whole system with respect to the symmetry axis of the accretion disk is set to $50^{\circ}$, which is representative of the orientation of typical "changing look" AGN (e.g., NGC 1365, Risaliti et al. 2013; Marin et al. 2013).

Throughout the different sections of the paper, we vary several additional parameters. First, the initial polarization vector of the source will be null (unpolarized photons, Sect. 2.2), uniformly saturated (Sect. 2.3), and then non-uniform (Sect. 2.4). The hydrogen column density of the gas cloud will vary from $10^{21}$ to and $10^{25} \mathrm{~cm}^{-2}$ to investigate a large panel of eclipsing schemes as reported by Markowitz et al. (2014).

\subsection{Impact of the cloud opacities}

Our initial study focused on a cloud with three different hydrogen column densities $\left(10^{21}, 10^{23}\right.$, and $\left.10^{25} \mathrm{~cm}^{-2}\right)$, shading an extended source radiating unpolarized photons. The cloud was set to be larger than the continuum source $\left(1.5 \times R_{\text {source }}\right)$, but it is slightly displaced from the observer's line of sight in order to only cover $50 \%$ of the disk at maximum obscuration. We investigated the passage of the gas clump over three energy bands: $1-5,7-12$, and $20-50 \mathrm{keV}$. We excluded the 5 to $7 \mathrm{keV}$ region owing to the strong, broad, fluorescent iron $\mathrm{K} \alpha$ emission $^{2}$ detected in a fraction of type-1 and intermediate AGN (Nandra et al. 2007). Such emission lines, which are unpolarized, would dilute the polarization signal. The $20-50 \mathrm{keV}$ band corresponds to the Compton hump, where Compton scattering is the most efficient way to enhance polarization. The polarization degree $P$ was integrated over the three energy bands in order to maximize the number count of photons and thus the detectability of the polarization signal.

We examine the time evolution of the X-ray continuum polarization resulting from an occultation event in Fig. 2. In terms of light curve variation, occultation of the primary source is seen through a sharp decrease in the flux, which reaches maximum attenuation when the cloud is at $\phi=90^{\circ}$, i.e. fully intercepting the observer's viewing angle, when obscuration is maximal. About half of the initial, soft X-ray flux is absorbed during occultation by a cloud with $n_{\mathrm{H}} \leq 10^{25} \mathrm{~cm}^{-2}$, which agrees with the XMM-Newton observation of Mrk 766 where a gas clump with $n_{\mathrm{H}} \sim 10^{23} \mathrm{~cm}^{-2}$ has eclipsed the central source, decreasing the flux and the $(6-10 \mathrm{keV}) /(2-5 \mathrm{keV})$ hardness ratio light curves of Mrk 766 by 50-60\% (Risaliti 2010). At $n_{\mathrm{H}} \sim 10^{23} \mathrm{~cm}^{-2}$, absorption effects are rather weak and mostly apparent in the soft X-ray spectrum. At higher energies, radiation propagates through the gas cloud with even less chance of absorption, and the three cloud models are hardly distinguishable.

An X-ray polarization continuum appears for all $n_{\mathrm{H}}$, energies and $\phi$, even when the cloud has not yet started occulting the source. Scattering onto the cloud gives rise to a polarization degree that decreases smoothly with the azimuthal position of the cloud when departing from $\phi=0^{\circ} \pm 180^{\circ}$. This is due to the scattering phase function of electron scattering that

2 Broad Fe K-shell fluorescence lines are due to X-rays radiated from a hot, optically thin corona of thermally distributed electrons, which is irradiating the disk surface from above. The neutral matter of the disk absorbs and partly reprocesses the flux into emission lines, where the strongest is the iron $\mathrm{K} \alpha$ line at $6.4 \mathrm{keV}$ (Fabian et al. 1989). The broadening of the line is ensured by general relativistic and Doppler effects shifting the line centroid as a function of the disk radius.
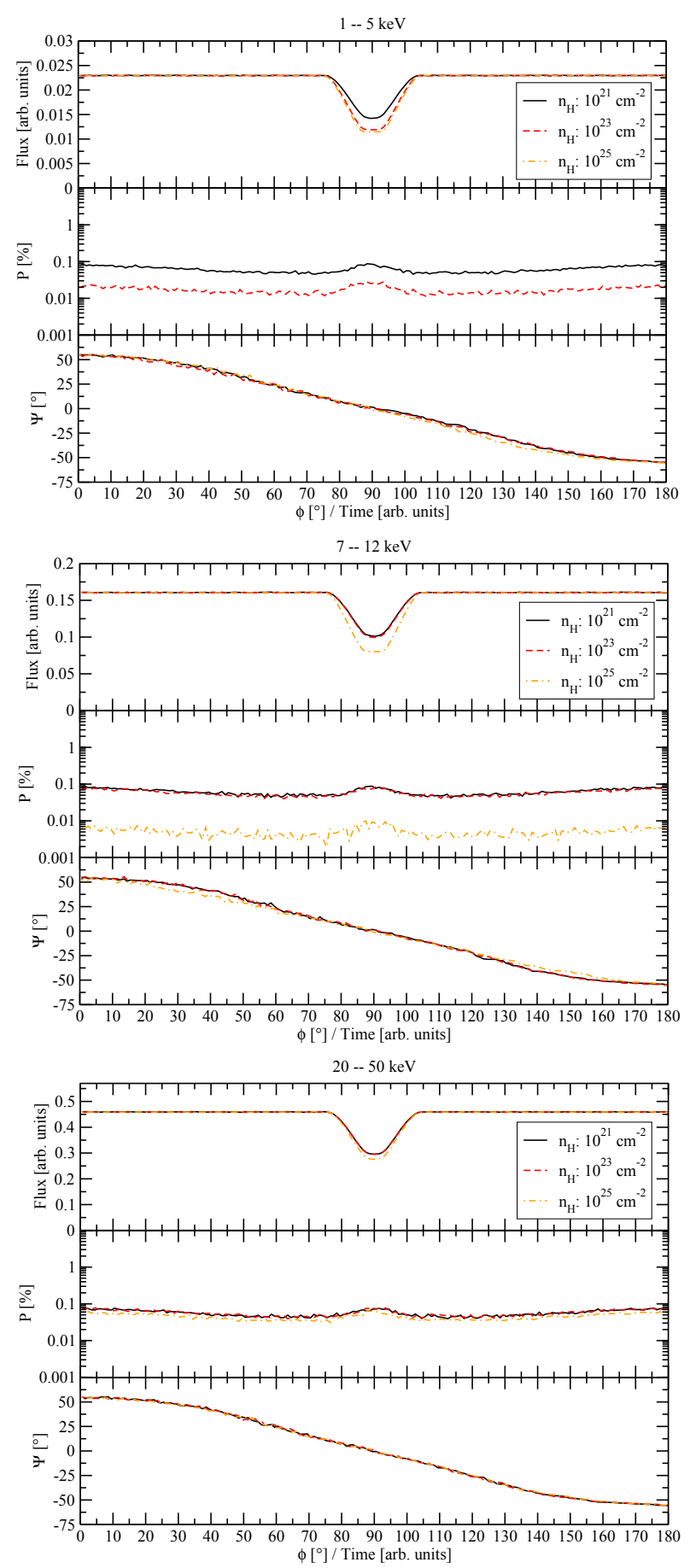

Fig. 2. Time evolution of the $\mathrm{X}$-ray continuum flux, polarization degree, and polarization position angle in the $1-5 \mathrm{keV}$ (top figure), $7-12 \mathrm{keV}$ (middle), and $20-50 \mathrm{keV}$ band (bottom) resulting from an occultation event. The eclipsing cloud covers $50 \%$ of the extended continuum source, and three different hydrogen column densities are considered for the gas (black: $10^{21} \mathrm{~cm}^{-2}$; red: $10^{23} \mathrm{~cm}^{-2}$; and orange: $10^{25} \mathrm{~cm}^{-2}$ ). Since that $P_{n_{\mathrm{H}}}=10^{25} \mathrm{~cm}^{-2}$ is too small, it does not appear in the top figure (see text).

is proportional to the cosine square of the scattering angle between the source, the cloud, and the observer. The maximum level of $P$ emerging from this configuration is $0.1 \%$, which by conventional detection capabilities, is rather low: $P$ depends on the photon energy, the position of the cloud, and the opacity of the gas. The transit of the cloud in front of the source does not cancel polarization because of 1) scattering of transmitted 
radiation penetrating the gaseous cloud; and 2) partial shielding of the primary source, preventing dilution of $P$ by unpolarized radiation. The resulting polarization at the occultation point is $\sim 0.1 \%$. Polarization is extremely low for very thick gas clouds in the $1-5 \mathrm{keV}$ band $\left(\sim 10^{-4} \%\right)$, since photons are heavily absorbed, allowing us to distinguish between Comptonthin and Compton-thick material. Polarization from dense gas clouds $\left(n_{\mathrm{H}}=10^{25} \mathrm{~cm}^{-2}\right)$ rises in the $7-12 \mathrm{keV}$ band but still is only $\sim 0.01 \%$. In the $20-50 \mathrm{keV}$ band, the three polarization signatures are similar owing to the high energies of the incoming photons, which face lower (energy-dependent) absorber scattering cross-sections. Soft X-ray polarization measurements are thus a better option for probing the opacity of an eclipsing gas cloud, while hard X-ray measurements would be more suitable for probing the intrinsic polarization properties of the source. Owing to the scattering-induced variation of $P$ and $\phi, X$-ray polarimetry predicts the passage of a cloud before it is detected with X-ray spectroscopy; however, such a signal is likely to be diluted by polarization by other LIL BLR clouds from the close environment of the central engine and thus be undetectable.

The polarization position angle $\psi$ resulting from reprocessing by the cloud brings additional, crucial information, where $\psi$ varies with the azimuthal phase of the cloud from $+50^{\circ}$ when $\phi=0^{\circ}$ to $-50^{\circ}$ at $\phi=180^{\circ}$. The sequence is monotonous and symmetric with respect to the maximal obscuration phase, where $\psi=0^{\circ}$. The maximum and minimum values of the polarization position angle trace the non axisymmetric setup of our model and are related to the observer's viewing angle. For a cloud rotating along the equatorial plane and an observer situated in the same plane, $\psi$ would be equal to $90^{\circ}$ (parallel polarization, measured with respect to the vertical axis of the system). All the gaseous clump models at all the energy ranges (even for a $10^{25} \mathrm{~cm}^{-2}$ cloud in the $1-5 \mathrm{keV}$ band) produce the same variation in the polarization position angle. Thus, if the initial photon flux radiated from AGN accretion disks is unpolarized, we expect to see variations in the measured polarization position angle before and after an occultation event.

\subsection{Occultation of a uniformly polarized source}

The polarization state of X-ray radiation emerging from AGN accretion disks was investigated by Chandrasekhar (1960) for the limiting case of $\tau \gg 1$. The intrinsic $P$ from an optically thick, ionized, scattering slab is expected to be due to multiple scattering and is inclination-dependent, with a maximum of $\sim 11 \%$ at edge-on inclinations. The polarization position angle is fixed and parallel to the disk symmetry axis, independent of the inclination. In this picture, the whole disk produces the same $P$ and $\psi$.

Following this approach, we now undertake the exploration of a grid of uniformly emitting accretion disks (partially) covered by distant gas clouds. Since the inclination of the system is $50^{\circ}$, the initial polarization is fixed to $2 \%$ with a parallel polarization position angle, which is a polarization state representative of a reprocessing disk at an intermediate inclination (Chandrasekhar 1960; Angel 1969; Lightman \& Shapiro 1975). We focus on the 1-5 keV band since 1) AGN primary flux in the soft X-ray band is larger than at harder regimes, thus enhancing detection capabilities; 2) most of the previous X-ray polarimetric mission concepts were designed to observe polarization in the soft band; and 3) we avoid the strong unpolarized Fe $\mathrm{L} \alpha_{1,2}$ $(\sim 0.705 \mathrm{keV})$ and $\mathrm{Fe} \mathrm{K} \alpha_{1,2}(\sim 6.397 \mathrm{keV})$ line complexes. We sample different hydrogen column densities (from $10^{22}$ to $\left.10^{25} \mathrm{~cm}^{-2}\right)$ and two different cloud sizes $\left(1.5\right.$ and $\left.3 \times R_{\text {source }}\right)$ with a covering factor of $50 \%$. All those cases are representative of the variable absorption along the observer's line of sight in the AGN sample monitored by the Rossi X-ray timing Explorer (RXTE) and presented by Markowitz et al. (2014). Their data were taken with the Proportional Counter Array (PCA) onboard the RXTE and limited to the $2-10 \mathrm{keV}$ band. They were sensitive to an absorption range of $\sim 10^{22}-10^{25} \mathrm{~cm}^{-2}$, with a detection peak situated in the $\sim 4 \times 10^{22}-2.6 \times 10^{23} \mathrm{~cm}^{-2}$ column density band.

The resulting grids are presented in Fig. 3. In terms of total flux, we find similar results to those in Fig. 2; i.e., light curve variations are governed by the hydrogen column density of the gas. Larger obscuring clouds with similar partial covering factor $(50 \%)$ tend to shade the continuum source longer and to create larger flux plateaus as they travel across the observer's line of sight. More significant differences appear in the polarization spectra: a $3 \times R_{\text {source }}$ obscuring clump strongly affect the continuum $P$ at $\phi=0^{\circ} \pm 180^{\circ}$. If, for a $1.5 \times R_{\text {source }}$ cloud with $n_{\mathrm{H}}=10^{22} \mathrm{~cm}^{-2}, P$ is affected by scattered radiation from the cloud surface (altering $P$ by less than $0.08 \%$ ), then bigger clouds change the polarization degree by $0.3 \%$ when occultation is maximum. This is due to the canceling contribution of parallel polarization from the disk and perpendicular polarization emerging from scattering, as seen in Fig. 2. The two polarization vectors cancel each other but, owing to the larger number of photons emitted from the disk, $P$ does not decrease to zero. With higher hydrogen column densities, this effect becomes less apparent since photons are predominantly absorbed rather than scattered. The impact of $n_{\mathrm{H}}$ and of the size of the eclipsing clump can also be seen in the polarization position angle spectra, because $\psi$ varies from $84^{\circ}$ to $94^{\circ}$ in the case of a Compton-thin absorber. This effect has better detection possibilities than observing a $<1 \%$ change in $P$ and could help to evaluate the size of the reprocessing medium.

It is therefore found that under special circumstances $\left(n_{\mathrm{H}} \leq\right.$ $10^{22} \mathrm{~cm}^{-2}$ and large absorbing cloudlets), an X-ray eclipse can significantly alter the observed polarization spectra. In those cases, the changes in $\psi$ could help to constrain the morphology and composition of the clump. However, for denser clouds partially covering an extended polarized source, the signal is dominated by the intrinsic polarization of the primary radiation.

\subsection{Shading a non-uniformly polarized emitting region}

According to Einstein's theory of gravity, the space time around compact objects is expected to be non-Euclidean, i.e. curved. Photons propagating close to the gravitational well are thus bent and move along geodesics. Computations of AGN disk irradiation by a hot corona situated above the disk surface, thereby producing X-ray photons via inverse Compton effect, showed that the resulting pattern is strongly anisotropic (Cunningham 1975). In addition, Connors et al. (1980) demonstrated that polarization features are also affected by general relativistic effects: the polarization vector of light is transported in parallel along the photons' null geodesic, while the degree of polarization is a scalar invariant. Therefore, if general relativistic effects are shaping the radiation from accretion disks, we expect to have a non-axisymmetric, reprocessed polarization pattern.

Similar to what has been done by Risaliti et al. (2011a) in spectroscopy, we now investigate the impact on polarization of partial disk obscuration by a distant gas cloud. To do so, we replace the uniformly emitting disk from our previous analysis by a toy-model disk composed of two different radiating parts (see Fig. 4). The receding red part of the accretion disk 

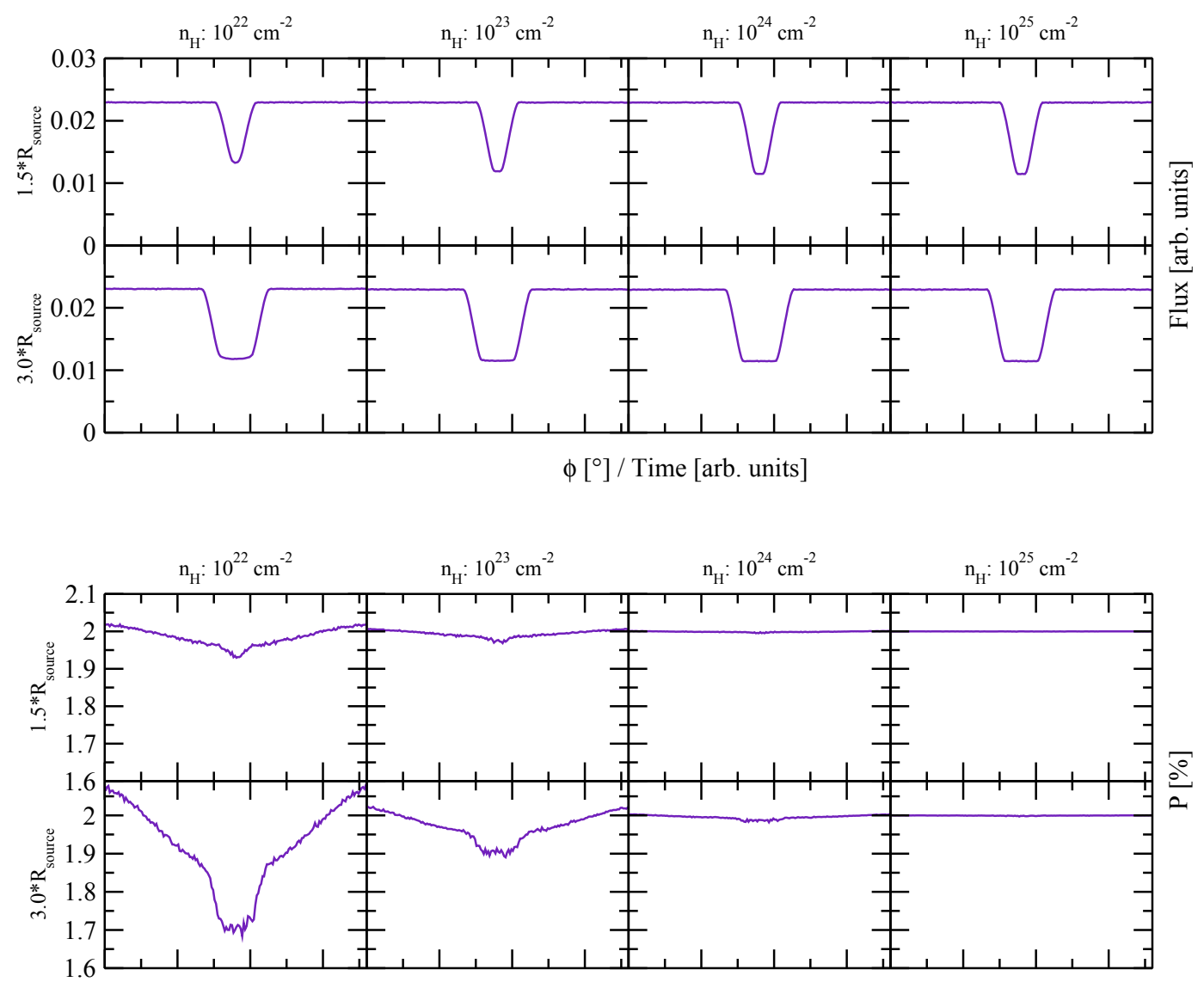

$\phi\left[^{\circ}\right] /$ Time [arb. units]

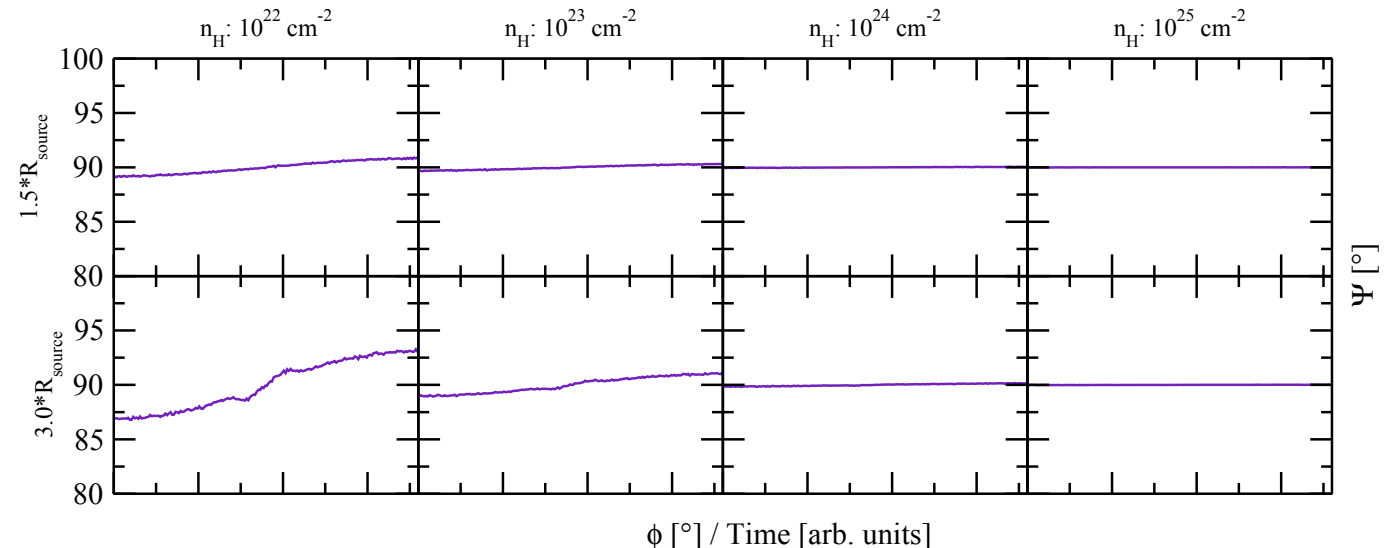

Fig. 3. Temporal evolution of the X-ray continuum flux (top figure), polarization degree (middle) and polarization position angle (bottom) in the 1-5 keV band resulting from the occultation of a primary source polarized parallely to the disk symmetry axis. The cloud hydrogen column density varies from $10^{22} \mathrm{~cm}^{-2}$ to $10^{25} \mathrm{~cm}^{-2}$ (columns) and its size relative to the emitting disk ranges from 1.5 to 3 (rows); nevertheless, the cloud's covering factor is set to $50 \%$.

is characterized by reprocessed radiation with an intrinsic polarization of $4 \%$ associated with a $52^{\circ}$ polarization position angle. The approaching blue part of the extended source emits photons with $P=2 \%$ and $\psi=90^{\circ}$. According to Cunningham (1975), the approaching and receding portions of the disk do not emit the same number count of photons per second. We set up three different models characterized by different luminosity ratios: $50 \%$ (blue part), $-50 \%$ (red part), 70\%-30\%, and 90\%-10\%. Here, $P$ and $\psi$ are arbitrary, but coherent representative values chosen from polarimetric simulations in strong gravity fields (Dovčiak et al. 2004, 2011; Schnittman \& Krolik 2010). Since the true
$\mathrm{X}$-ray polarization signal is likely to vary from object-to-object due to a different black hole mass, disk inclination, or spin, we thus investigate a generic case.

Figure 5 shows the variation in flux and polarization during an X-ray eclipse by a $10^{24} \mathrm{~cm}^{-2}$ gas cloud (a value similar to the $3.5 \times 10^{23} \mathrm{~cm}^{-2}$ hydrogen column density used by Risaliti et al. 2011a) covering about $90 \%$ of the emitting accretion disk (ibid.). The shape of the flux profile during an occultation event is symmetric around $\phi=90^{\circ}$ when the two parts of the disk emit the same number of photons, but departs from symmetry when the radiation pattern is affected by relativistic beaming. 


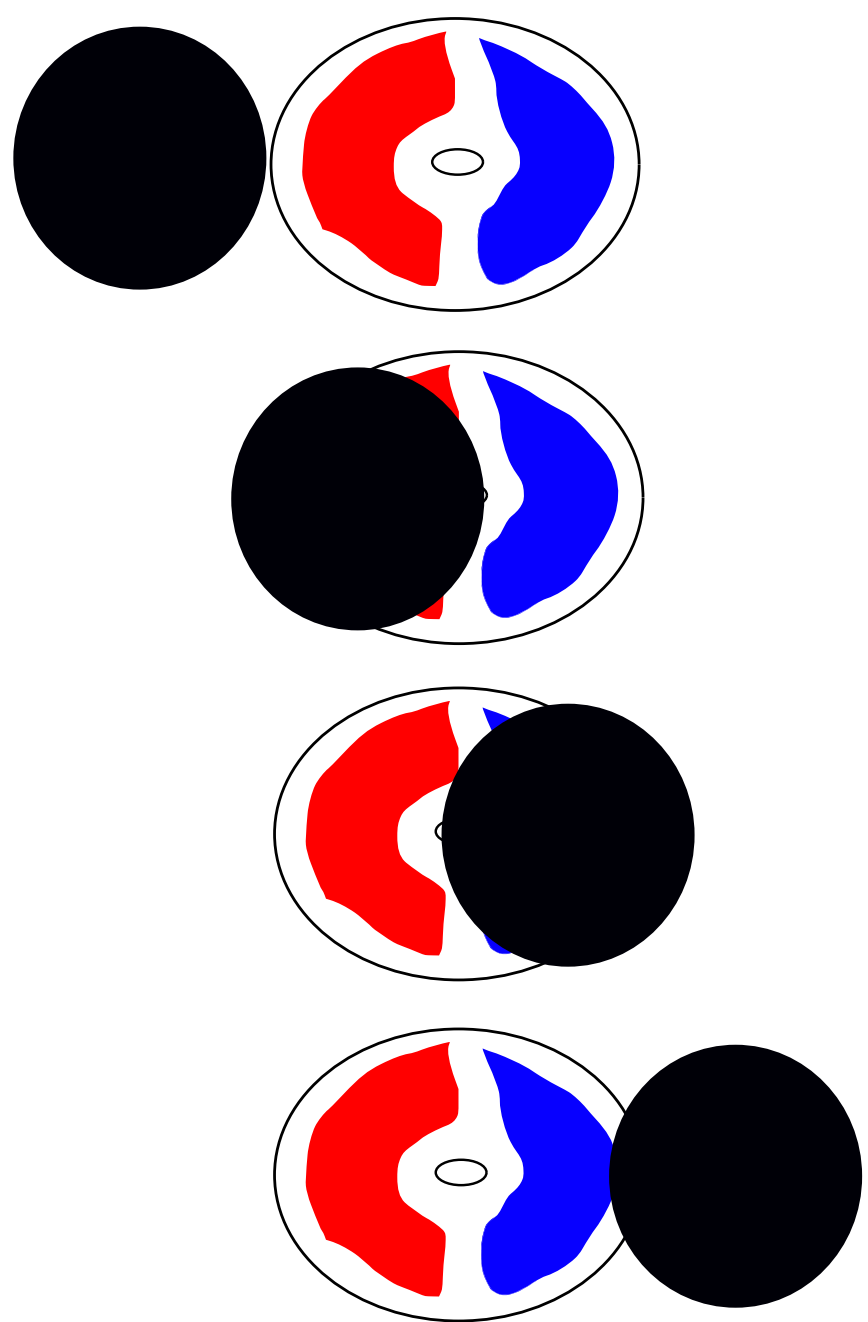

Fig. 4. Illustration of an obscuring cloud shading different parts of an inclined accretion disk rotating in the clockwise direction. The blue part of the accretion disk corresponds to the maximum intensity flux caused by relativistic beaming and shows a different integrated polarization state than the red part of the sketch.

Covering the approaching (blue) part of the disk strongly affects the number of photons received by the observer and results in an asymmetric pattern, as expected by Risaliti et al. (2011a). Maximum asymmetry appears for the 90-10\% model.

Polarization signatures show the most dramatic variations due to the successive covering and uncovering of the two halves of the disk. When the disk is not obscured, the net polarization degree and polarization position angle are integrated in a way that depends on the emissivity of the approaching or receding regions. For a disk with a 50\%-50\% illumination ratio, the total $P$ is equal to $\sim 2.5 \%\left(\psi=63^{\circ}\right)$, while for a $90 \%-10 \%$ model, $P \sim 2.0 \%\left(\psi=84^{\circ}\right)$. Since the receding part of the disk pattern is set to emit minimum fluxes, it is natural that the integrated polarization degree and angle are dominated by the intrinsic polarization of the Doppler-boosted part. As soon as the red part of the accretion disk, which is responsible for the $4 \%$ polarization associated with $\psi=52^{\circ}$, becomes obscured, the net polarization decreases smoothly to $2 \%$ for all models. The total polarization position angle follows the same trend, switching to parallel polarization. Uncovering the receding red part of the disk and starting to cover its approaching blue part imprints polarization in a way that depends more on the model. Here,

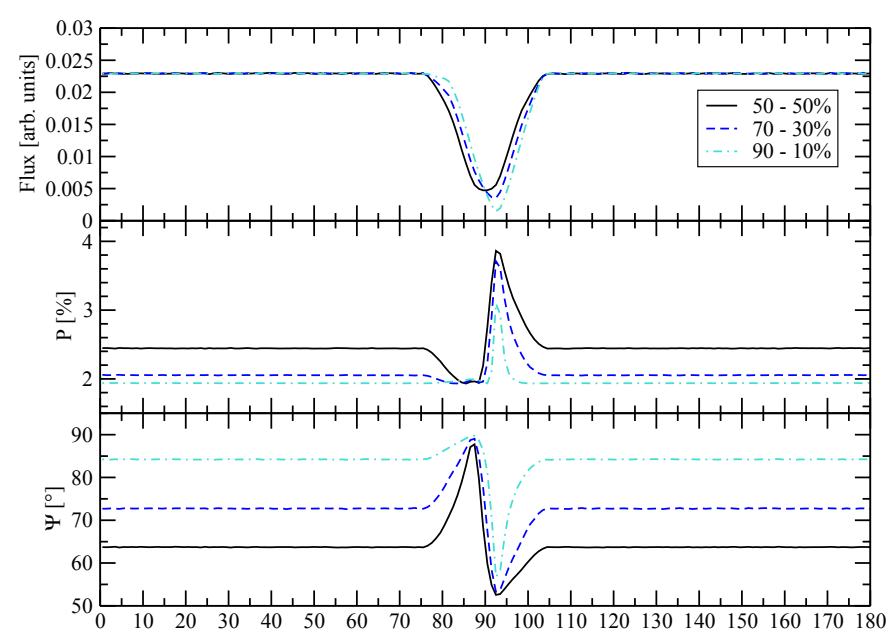

Fig. 5. Time evolution of the X-ray continuum flux, polarization degree, and polarization position angle in the $1-5 \mathrm{keV}$ band, resulting from the shading of different parts of a non-uniformly polarized emitting disk. The cloud is slightly smaller than the continuum source and has a hydrogen column density of $10^{24} \mathrm{~cm}^{-2}$. Three different emission ratios are considered with $90 \%$ (turquoise), $70 \%$ (blue), or 50\% (black) of the total luminosity being emitted by the blue part of the reprocessing disk.

$P$ rises up to $4 \%$ and $\psi$ decreases to $52^{\circ}$ for a $50 \%-50 \%$ scenario, but a model with higher approaching emissivities (e.g., $90 \%-10 \%$ ) sees smaller $P$ variations. This is due to a large number of transmitted and scattered radiation penetrating the gaseous cloud and altering the net polarization position angle. This effect can be seen in the polarization position angle, where the same model is found not to reach exactly $52^{\circ}$ owing to intrinsically polarized photon transmitted through the clump and partial coverage. Once the cloud uncovers the approaching half, $P$ and $\psi$ return to their original, integrated values. It is noteworthy that the variation in X-ray polarization during an eclipsing event is found to be continuous, not sharp, forming a P Cygni type profile $^{3}$ that can be symptomatic of general relativistic effects or, at least, of non-axisymmetric irradiation from the accretion disk.

\section{Discussion}

In agreement with Risaliti et al. (2011a), we have shown that X-ray eclipses affect the resulting fluxes observed by a distant observer, but we also provided the first time-dependent estimation of the polarimetric signatures we could expect from obscuration events. We stress that our baseline model provides a simple but good indicator of the polarization that a future X-ray polarimeter could observe, and we now discuss more complex cloud models and the possibility of adapting our work to extraterrestrial (exoplanet) research.

\subsection{Comet-like gas structures}

Our X-ray polarimetric simulations showed that Compton-thin and Compton-thick eclipses detected in "changing look" AGN give different polarimetric signals according to the intrinsic polarization of the source and the size of the gas clump. The

3 P Cygni profiles are spectroscopic features usually detected in stel-
lar winds and characterized by strong emission lines from an expand-
ing shell with corresponding blueshifted absorption lines from material ing shell with corresponding blueshifted a
moving away from the star and toward us. 
geometry of the obscuring cloud we used is spherical, but a different structure can also alter the resulting polarization spectra. From their time-resolved spectral analysis of Mrk 766, Risaliti et al. (2011b) find hints of a more complex structure of the eclipsing cloud than a spherical clump, where strong ionization gradients point toward a comet-like geometry. In their picture, the gas clumps are structured as follows: a dense, cold core of absorbing matter is surrounded by low-density, higher ionization layers of gas. The motion of the cloud is responsible for a Compton-thin cometary tail of gas extracted from the highdensity cloudlet head. The resulting morphology of the occulting medium thus departs from a spherically-symmetric model.

Since polarization is sensitive to any departure from symmetry, such a configuration is expected to increase the resulting $P$ of our simulations owing to additional Compton scattering within the cometary tail of the cloud. On the other hand, $\psi$ might change according to Fig. 3: the low-density tail of the clump may be responsible for a smooth rotation of the polarization angle after the passage of the cold head, allowing variations in polarization in a larger time frame. In addition, McNamara et al. (2008) shows that, for the accretion columns in $\mathrm{mCVs}$, the resulting polarization is sensitive to the density structure of the model. In a similar way, a comet-like cloud may leave a particular imprint on polarization that would allow us to probe the matter and temperature stratification of those eclipsing media.

\subsection{Application to other astronomical sources}

The Keplerian orbit of a point-like object around a radiation source is reminiscent of another astrophysical domain: planetary and exo-planetary research. By a simple analogy and dimension magnification, it is easy to consider that our gas clump can be representative of an exoplanet transiting in front of its parent star. We saw in Sect. 2.2 that when a gas clump revolves around its irradiating disk, the scattering angle changes and the resulting polarization varies. Scattering-induced polarization results from reprocessing of radiation onto the surface of the cloud, leading to polarization degrees of about $0.1 \%$ and time-dependent $\psi$. In this case, X-ray polarimetry could be used to detect the rotation of a planet around its host.

In the optical domain, the first direct detection of an exoplanet by polarimetric techniques was done by Berdyugina et al. (2008), who targeted HD 189733b, a transiting exoplanet belonging to the hot Jupiter classification, known to have a very short period (i.e., a small orbit), which makes it a valuable candidate for polarimetric detections. From the Stokes parameters $q$ and $u$ (normalized to the total flux), Berdyugina et al. (2008) inferred the size of the extended atmosphere to be on the order of $1.5 \pm 0.2 R_{\text {Jupiter }}$, with a lower limit of the albedo as 0.14 . In addition, the observed polarization variability was used to corroborate the previously estimated orbital period of the planet, inclination, eccentricity, and orientation of the orbit.

Although transparent in the optical band, the atmosphere of exoplanets is opaque at very soft X-ray energies, blocking most of the star's emission (Poppenhaeger et al. 2013). A model of a gas clump with $n_{\mathrm{H}} \leq 10^{23} \mathrm{~cm}^{-2}$ and a radius smaller than the extended corona of the star could then be used as a first-order approximation to evaluate the resulting polarization from HD 189733b. The coronal X-ray fluxes of solar-type stars originating in bremsstrahlung emission and peaking in the 1-2 keV band, we intend to run new Monte Carlo simulations with adapted sizes and geometries to explore such a path (Marin $\&$ Grosso, in prep.). Since ordinary bremsstrahlung from low$Z$ material is unpolarized (Dolan 1967), the scattered light from
HD 189733 b should not be diluted by any intrisic polarization from its parent star and, according to Fig. 2, $P$ will be non-null. We note, though, that the luminosity of the parent star may be an issue for a future X-ray polarimetric detection of a hot Jupiter since the luminosity of, for example, the primary component of HD 189733 in the $0.25-2 \mathrm{keV}$ band is only on the order of $L_{\mathrm{X}} \sim$ $10^{28} \mathrm{erg} \mathrm{s}^{-1}$ (Poppenhaeger et al. 2013).

\section{Conclusions}

This paper has shown how X-ray polarimetry, particularly in the soft, $1-5 \mathrm{keV}$ band, can be used to probe occulting events in AGN. We demonstrated that the resulting polarization spectra strongly depend on the initial state of continuum radiation.

In the case of an extended disk emitting unpolarized photons, a residual polarization degree appears to be due to scattering onto the cloud surface. Its polarization degree is low $(P \ll 1 \%)$, and its polarization position angle depends on both the inclination of the system and on the azimuthal position of the cloud. For intermediate inclinations, $\psi$ rotates from $+50^{\circ}$ to $-50^{\circ}$, with a resulting polarization position angle perpendicular to the symmetry axis of the disk when the cloud is situated between the source and the observer.

Looking at polarized primary sources, important differences identify uniformly and non-uniformly emitting disks. When the source region radiates photons with a polarization state independent of the emission point, X-ray eclipses weakly impact $P$ and $\psi$ for Compton-thick clouds. In the case of clumps with $n_{\mathrm{H}} \leq 10^{22} \mathrm{~cm}^{-2}, P$ and $\psi$ become time-dependent but the net variations are rather small $\left(\Delta P<1 \%\right.$ and $\left.\Delta \psi<10^{\circ}\right)$, even when considering large obscuring clouds. Unfortunately, such weak polarization variations are probably not detectable in the context of the present polarimetric mission projects.

Major differences are spotted for non-uniformly polarized emitting regions predicted by general relativity. In this case, the receding and approaching parts of the accretion disk are characterized by different intrinsic polarization, and the net polarization depends on the emissivity of the two parts. The resulting polarization variations due to the successive covering and uncovering of the two halves of the disk are then very distinctive: $P$ can vary by a few percent, and $\psi$ can switch from parallel to intermediate or perpendicular polarization according to the initial parametrization. P Cygni type profiles are found to characterize the net polarization in our toy model, and similar results are expected for more complex, polarized illumination patterns. Interestingly, several percents of variation could be in the reach of a large collecting area satellite equipped with a state-of-the-art polarimetric instrument if targeting a luminous object.

As a result, time-dependent polarimetry may open a new and distinctive window for probing general relativistic effects arising close to compact objects. In addition, polarization would help to remove the following degeneracy: Is the intensity fluctuation intrinsic to the source or due to an eclipsing event? If intrinsic, more chaotic variation of $P$ and/or $\psi$ are expected.

Acknowledgements. This research has been partially supported by the European Union Seventh Framework Program (FP7/2013-2017) under grant agreement No. 312789, StrongGravity. The authors would like to acknowledge the additional support from the grants COST-CZ LD12010 and COST Action MP1104, as well as the Academy of Sciences of the Czech Republic for its hospitality. F.M. is grateful to Nicolas Grosso and Delphine Porquet for their valuable suggestions.

\section{References}

Angel, J. R. P. 1969, ApJ, 158, 219

Berdyugina, S. V., Berdyugin, A. V., Fluri, D. M., \& Piirola, V. 2008, ApJ, 673, L83 
Chandrasekhar, S. 1960, Radiative transfer (New york: Dover) Connors, P. A., Stark, R. F., \& Piran, T. 1980, ApJ, 235, 224 Cunningham, C. T. 1975, ApJ, 202, 788

Dolan, J. F. 1967, Space Sci. Rev., 6, 579

Dovčiak, M., Karas, V., \& Matt, G. 2004, MNRAS, 355, 1005

Dovčiak, M., Muleri, F., Goosmann, R. W., Karas, V., \& Matt, G. 2011, ApJ, 731, 75

Fabian, A. C., Rees, M. J., Stella, L., \& White, N. E. 1989, MNRAS, 238, 729

Goosmann, R. W., \& Gaskell, C. M. 2007, A\&A, 465, 129

Goosmann, R. W., \& Matt, G. 2011, MNRAS, 415, 3119

James, C. H., Ramsay, G., Mark Cropper, M. C., \& Branduardi-Raymont, G. 2002, MNRAS, 336, 550

Lee, H. W. 1994, MNRAS, 268, 49

Lee, H.-W., Blandford, R. D., \& Western, L. 1994, MNRAS, 267, 303

Lightman, A. P., \& Shapiro, S. L. 1975, ApJ, 198, L73

Marin, F., Goosmann, R. W., Gaskell, C. M., Porquet, D., \& Dovčiak, M. 2012, A\&A, 548, A121

Marin, F., Porquet, D., Goosmann, R. W., et al. 2013, MNRAS, 436, 1615

Markowitz, A. G., Krumpe, M., \& Nikutta, R. 2014, MNRAS, 439, 1403

McNamara, A. L., Kuncic, Z., \& Wu, K. 2008, MNRAS, 386, 2167
Nandra, K., O’Neill, P. M., George, I. M., \& Reeves, J. N. 2007, MNRAS, 382, 194

Netzer, H., \& Laor, A. 1993, ApJ, 404, L51

Peterson, B. M. 2001, in Advanced Lectures on the Starburst-AGN, Proc. Conf., Puebla, Mexico, eds. I. Aretxaga, D. Kunth, \& R. Mújica (Singapore: Work Scientific), 3

Poppenhaeger, K., Schmitt, J. H. M. M., \& Wolk, S. J. 2013, ApJ, 773, 62 Risaliti, G. 2010, in IAU Symp., 267, eds. B. M. Peterson, R. S. Somerville, \& T. Storchi-Bergmann, 299

Risaliti, G., Elvis, M., \& Nicastro, F. 2002, ApJ, 571, 234

Risaliti, G., Miniutti, G., Elvis, M., et al. 2009, ApJ, 696, 160

Risaliti, G., Nardini, E., Elvis, M., Brenneman, L., \& Salvati, M. 2011a, MNRAS, 417, 178

Risaliti, G., Nardini, E., Salvati, M., et al. 2011b, MNRAS, 410, 1027

Risaliti, G., Harrison, F. A., Madsen, K. K., et al. 2013, Nature, 494, 449

Schnittman, J. D., \& Krolik, J. H. 2010, ApJ, 712, 908

Suganuma, M., Yoshii, Y., Kobayashi, Y., et al. 2006, ApJ, 639, 46

Ulrich, M.-H., Maraschi, L., \& Urry, C. M. 1997, ARA\&A, 35, 445

White, N. E., \& Holt, S. S. 1982, ApJ, 257, 318

Wills, B. J., Laor, A., Brotherton, M. S., et al. 1999, ApJ, 515, L53 